\title{
Experiencias en la aplicación de tabletas en Secundaria
}

\section{Experiences Using Tablets in Secondary School}

Félix Buendía García ?, José V. Benlloch Dualde ?, Inmaculada Zahonero Viana 2, Andrés Cubel Barea $^{2}$

1 Departamento de Informática de Sistemas y Computadores, Universitat Politècnica de València, Valencia. España. \{fbuendia, jbenlloc\}@ disca.upv.es

${ }^{2}$ Colegio Asunción de Ntra. Señora Benaguasil, Benaguasil, Valencia. España \{inma.zahonero, andres.cubel\}@asuncionbenaguasil.es

\begin{abstract}
Resumen
El objetivo de este trabajo consiste en presentar una serie de reflexiones sobre el uso de dispositivos tablet (tabletas) en el ámbito de la Enseñanza Secundaria. Este tipo de dispositivos viene utilizándose en diversos centros educativos repartidos por el estado español, principalmente en Educación Primaria, y pese a su potencial innovador y las ventajas tecnológicas que aportan, también aparecen dificultades cuando se busca una implantación efectiva de los mismos. Por un lado, en el trabajo se plantean ciertas cuestiones sobre el aprovechamiento didáctico de estos dispositivos y las necesidades o requisitos que ello implica. Por otra parte, se trata de extraer una serie de recomendaciones para una aplicación efectiva de este tipo de dispositivos. Para ello, se cuenta con la experiencia de un centro educativo a lo largo de varios cursos en la aplicación de tales tecnologías. A partir de dicha experiencia se analizan los resultados obtenidos y se pretende dar una nueva perspectiva en el uso didáctico de las tabletas.
\end{abstract}

\section{Palabras Clave}

Tableta; educación secundaria; itinerario educativo; método didáctico.

\begin{abstract}
The aim of the current work is introducing some findings about the use of tablet devices in the context of Secondary Education. This kind of devices is used in many schools, all over the Spanish country, and particularly in Primary Education. In spite of their innovation potential and their technical contributions, some difficulties can be found to implement tabletbased scenarios in an effective way. On the one hand, the work deals with some issues about the didactical deployment of such devices together with their educational requirements. On the other hand, some recommendations are provided to support an effective use of these devices. In this sense, an experience about the application of this kind of technology within an educational institution throughout several courses is reported. From this experience, the gathered results are analyzed and a new perspective in the didactical use of tablets is depicted.
\end{abstract}

\section{Keywords}

Tablet; secondary education; learning path; didactic method. 


\section{Introducción}

Las tecnologías móviles están presentes en múltiples ámbitos de la educación, desde los niveles más básicos hasta la enseñanza universitaria y permanente ("lifelong learning"). Una de las tecnologías con mayor impacto es la asociada a los dispositivos "tablet" (tabletas en adelante) que pueden identificarse como una especie de ordenador, cuya característica principal es la de tener una pantalla táctil donde se realizan la mayoría de acciones. El informe Horizon (Johnson, Adams y Cummins, 2012) ya reconocía el papel de este tipo de dispositivos y su implantación a corto plazo en el ámbito universitario, e informes como el UNIR (2013) o Ditendria (2015) indican su grado de penetración a nivel del estado español. Un estudio de la OEI (Marés, 2012) señalaba las oportunidades y desafíos que suponía la introducción de tabletas en educación.

El presente trabajo invita a una serie de reflexiones sobre su uso más allá del debate meramente tecnológico y presenta diversas consideraciones sobre su aplicación didáctica en el ámbito de la enseñanza secundaria. Las tabletas han sido utilizadas en diversos proyectos e iniciativas, principalmente asociadas al ámbito de la Educación Primaria. Podemos citar algunos ejemplos, como el programa PizarraDigital, que combinaba este recurso con el uso de dispositivos TabletPC (Ferrer, Belvis y Pamies, 2011) o el proyecto Dedos (CITA, 2011). Sin embargo, el contexto de la Educación Secundaria introduce una serie de factores diferenciadores en el uso de las tabletas respecto a otros ámbitos. En primer lugar, la infraestructura disponible en los centros educativos plantea problemas de gestión de los dispositivos empleados o su propia conectividad a Internet, derivados de una mayor concentración de alumnos. En segundo lugar, la procedencia de alumnado desde diversos centros, y con competencias digitales diferentes, junto con las propias peculiaridades de los alumnos en estas edades, suponen una dificultad añadida. También hay que citar la falta de aplicaciones específicas y la escasa formación del profesorado en el uso de estas tecnologías. Este tipo de problemáticas ha sido analizada por Pere Marqués (2014), en una investigación realizada con 39 centros, donde destaca la falta de tiempo y de preparación del profesorado como factores que más dificultan la aplicación de las tabletas. Todo ello conduce a la necesidad de establecer un estudio sobre las experiencias de aprendizaje basadas en el uso de tabletas y sus aplicaciones didácticas. A partir de este objetivo principal se plantean otros de tipo secundario, como la propuesta de enfoques y técnicas de trabajo con esta clase de recursos tecnológicos, o la presentación de situaciones que pueden desaconsejar su uso en determinados contextos. El resto del artículo se estructura en las siguientes secciones, empezando por el Método utilizado para analizar las experiencias de aprendizaje objeto de estudio. La tercera sección muestra algunos de los Resultados obtenidos tras el estudio y en la siguiente sección se plantea una Discusión de los mismos. Finalmente, se indican las principales Conclusiones derivadas del trabajo realizado. 


\section{Marco teórico}

En este apartado se describen los aspectos metodológicos que intervienen en el análisis de las experiencias vinculadas con la aplicación de tabletas en el ámbito de la Educación Secundaria. En primer lugar, se exponen una serie de enfoques y técnicas docentes orientadas al uso de este tipo de tecnologías. A continuación, se plantea el contexto de aplicación en un ámbito específico correspondiente a un centro educativo concreto y, por último, se presenta el procedimiento de trabajo puesto en práctica.

\subsection{Uso didáctico de las tabletas}

En este punto se analizan una serie de aspectos que condicionan la aplicación o uso didáctico de las tabletas. Para ello se plantean diversos enfoques docentes (De la Herrán Gascón, 2008) desde la tradicional "lección magistral" (expositiva) hasta los métodos orientados al llamado "aprendizaje activo". En la Tabla 1 se muestran algunas de las técnicas y mecanismos para su aplicación en un entorno tecnológico.

\begin{tabular}{|c|c|}
\hline Técnicas & Aplicación tecnológica \\
\hline Expositiva & Uso del proyector o pizarra digital para presentar contenidos multimedia. \\
\hline Expositiva interactiva & $\begin{array}{l}\text { Los contenidos presentados en el proyector se pueden enriquecer con material que permita al } \\
\text { profesor interactuar con el mismo. }\end{array}$ \\
\hline $\begin{array}{l}\text { Expositiva con } \\
\text { participación }\end{array}$ & $\begin{array}{l}\text { La presentación de contenidos o recursos asociados puede complementarse con la } \\
\text { intervención del alumnado a través de tabletas. }\end{array}$ \\
\hline $\begin{array}{l}\text { Trabajo individual } \\
\text { presencial }\end{array}$ & $\begin{array}{l}\text { El alumno realiza actividades de forma autónoma mediante la revisión de contenidos o la } \\
\text { resolución de tareas sin necesidad de conexión a Internet. }\end{array}$ \\
\hline $\begin{array}{l}\text { Trabajo individual } \\
\text { "online" }\end{array}$ & $\begin{array}{l}\text { Las actividades realizadas por el alumno en su tableta pueden tener una conexión de forma que } \\
\qquad \text { se reciban respuestas a cuestionarios o tareas. }\end{array}$ \\
\hline $\begin{array}{l}\text { Trabajo individual } \\
\text { con comunicación }\end{array}$ & $\begin{array}{l}\text { El alumno puede presentar un trabajo realizado de forma individual a través del proyector o } \\
\text { pizarra o desde su tableta conectada a estos dispositivos. }\end{array}$ \\
\hline $\begin{array}{l}\text { Trabajo grupal } \\
\text { expositivo }\end{array}$ & $\begin{array}{l}\text { Se puede plantear una actividad para que sea realizada por un grupo de alumnos mediante la } \\
\text { asignación de tareas individuales. }\end{array}$ \\
\hline $\begin{array}{l}\text { Trabajo grupal } \\
\text { cooperativo }\end{array}$ & $\begin{array}{l}\text { El trabajo planteado para un grupo de alumnos puede requerir la comunicación entre } \\
\text { ellos y la consecución de un fin común mediante tabletas. }\end{array}$ \\
\hline $\begin{array}{l}\text { Trabajo grupal } \\
\text { interactivo }\end{array}$ & $\begin{array}{l}\text { El propósito del trabajo de grupo puede consistir en elaborar un producto con el que } \\
\text { puedan interactuar el resto de alumnos. }\end{array}$ \\
\hline
\end{tabular}

Tabla 1. Técnicas de trabajo con recursos tecnológicos

Las técnicas de tipo "expositivo" se asocian a actividades docentes donde el profesor tiene un papel preponderante, aunque también se puede promover la participación del alumnado. El uso de 
dispositivos tecnológicos tales como el proyector o la pizarra digital puede servir para dar soporte a la exposición de contenidos en diferentes formatos (texto, audio, imágenes o vídeo) o que incluyan aplicaciones para mostrar simulaciones (p. e. el movimiento del planeta Tierra) o incluso juegos asociados a la materia expuesta. La tableta podría utilizarse, por parte del profesor que expone los contenidos, como un mecanismo que facilite el trabajo con estos, más allá de la típica interacción con el ordenador que controla el proyector, a través del teclado/ratón. Incluso permitiría una mayor movilidad al profesor, que podría desplazarse por la clase, observando las reacciones del alumno. Este también podría aprovechar la tableta para participar durante la sesión y poder realizar preguntas de forma anónima o anotar algún comentario sobre la exposición.

En el caso del trabajo individual o autónomo, se puede diferenciar aquel que realiza el alumno de forma presencial, ya sea en clase o en un laboratorio, pero que no requiere una conexión a Internet, del trabajo que requiere este tipo de conexión. Por ejemplo, la tableta podría utilizarse para que el alumno consulte ciertos contenidos o realice determinadas tareas de manera "offline" sin necesidad de transmitir los resultados de tales actividades. La modalidad o técnica de trabajo "online" permitiría registrar de manera inmediata tales resultados para su posterior evaluación.

Para las técnicas de trabajo grupal también pueden distinguirse varias modalidades según el objetivo de la actividad a realizar. En el caso más simple se trataría de repartir el trabajo entre los alumnos mediante la asignación de tareas individuales donde cada uno de ellos pueda trabajar de forma autónoma con su tableta. Un trabajo cooperativo podría consistir, por ejemplo, en la elaboración de un audiovisual sobre el funcionamiento del sistema solar y ello requeriría mecanismos de comunicación y compartición de resultados (vía Google Drive o similar). Finalmente, la preparación de un trabajo que permita ciertas características de interacción exigiría el uso de herramientas más sofisticadas (pizarra virtual, conferencia web...).

\subsection{Contexto de aplicación}

Las técnicas descritas en el apartado anterior se han aplicado en un contexto real correspondiente a un centro educativo de la localidad de Benaguasil (Valencia). Se trata de un colegio diocesano que, recientemente, ha cumplido 48 años. Actualmente está constituido por dos líneas educativas, desde Educación Infantil (2 años), hasta $4{ }^{\circ}$ de Enseñanza Secundaria Obligatoria (ESO). Además, se imparten dos Ciclos Formativos de Grado Medio (CFGM) en las ramas de Gestión Administrativa y Comercio. El centro cuenta en la actualidad con unos 800 alumnos, 250 en las etapas de ESO y CFGM, y 550 en las etapas de Infantil y Primaria. Un 10\% de los alumnos del centro son alumnos inmigrantes, y un $11 \%$ son alumnos con necesidades educativas especiales, necesidades de apoyo logopédico o compensación educativa. 
El centro inició su experiencia en el uso de tabletas en el año 2012, naciendo como resultado de un proyecto educativo denominado EDUCABE (Arrue, 2012). En su primer año de implantación se aplicó a $5 .^{\circ}$ de Primaria y $1 .^{\circ}$ y $3 .^{\circ}$ de ESO, y posteriormente se incorporó a $6 .^{\circ}$ de Primaria y $2 .^{\circ}$ y $4 .^{\circ}$ de ESO. En la actualidad dicho uso se ha extendido a un total de 350 alumnos de tercer ciclo de Educación Primaria y todos los cursos de ESO. La Tabla 2 muestra los datos numéricos más relevantes de la experiencia, referidos al curso 2014-15. Un dato a resaltar es que la media de edad entre los profesores de Primaria era casi 10 años inferior a la de los de Secundaria. Respecto el número de alumnos por grupo resulta muy similar entre los ámbitos de Educación Primaria y Secundaria.

\begin{tabular}{|c|c|c|}
\hline Niveles & Primaria & Secundaria \\
\hline N. ${ }^{\circ}$ alumnos & 100 & 250 \\
\hline N. ${ }^{\circ}$ profesores & 7 & 18 \\
\hline Media edad profesorado & 38,25 & 47,18 \\
\hline Grupos por curso & 2 & 2 \\
\hline Grupos totales & 4 & 8 \\
\hline Media alumnos/grupo & 25,5 & 24,4 \\
\hline
\end{tabular}

Tabla 2. Datos de usuarios de proyecto EDUCABE (2014-15)

El proyecto EDUCABE plantea un enfoque metodológico común para los alumnos desde su incorporación al centro educativo hasta el final de la educación secundaria obligatoria. Inicialmente, los materiales didácticos a impartir en el aula fueron elaborados por el profesor, adaptándose en todo momento a las circunstancias y necesidades de aprendizaje real de sus respectivos alumnos.

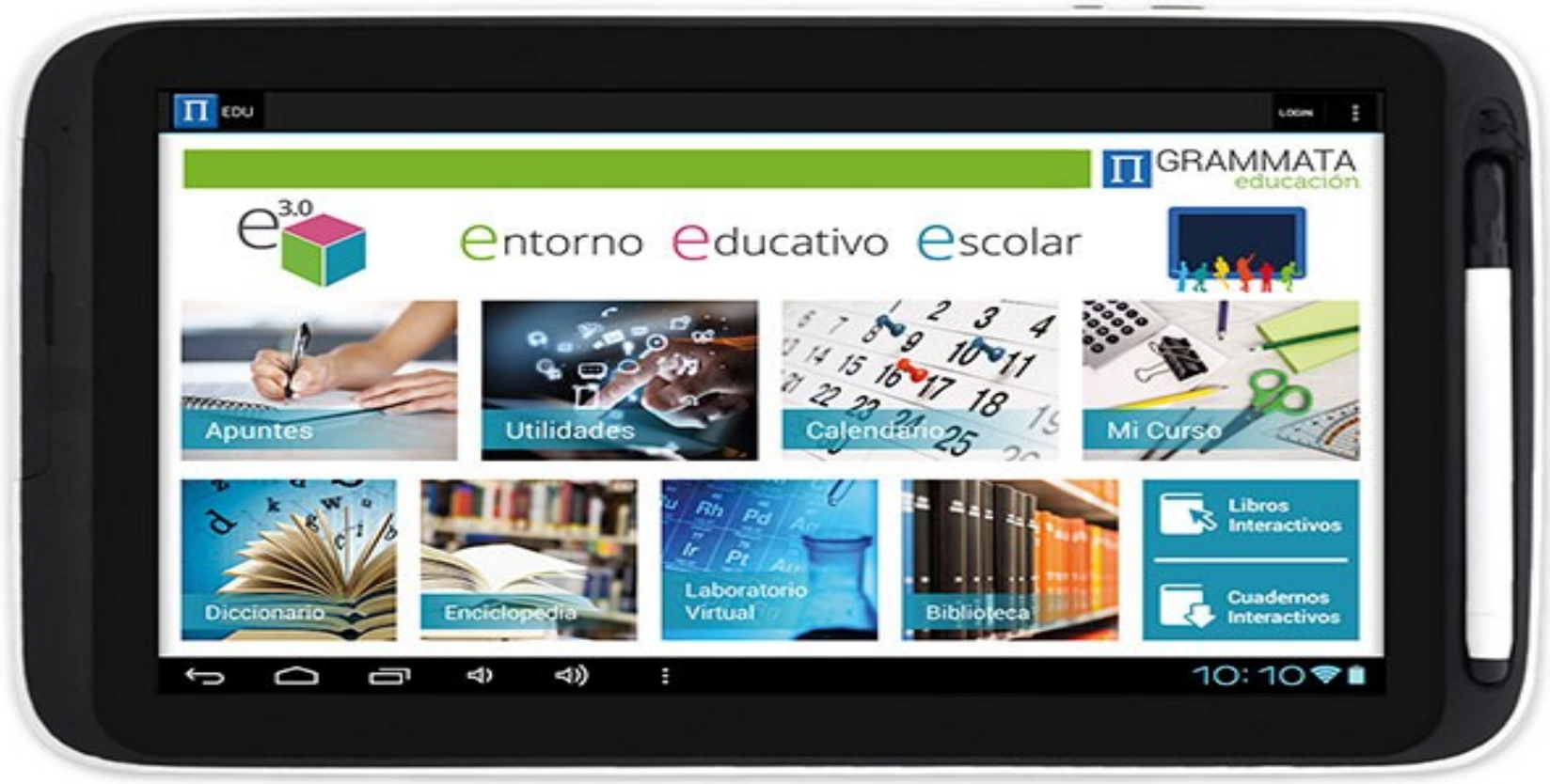

Figura 1. Modelo Tableta Grammata Papyre 1010

Durante el curso 2014-2015 se introdujo como novedad, para los cursos de $5 .^{\circ}$ y $6 .^{\circ}$ de Primaria, el 
uso de los materiales didácticos de una editorial, por lo que en este caso se combinan en las tabletas dichos materiales didácticos interactivos con los materiales elaborados propios. Desde el inicio del proyecto los alumnos sustituyeron el libro de texto por una tableta Grammata cuyo modelo Papyre 1010 se muestra en la Figura 1. En posteriores cursos se incluyeron nuevos modelos (Papyre 1015) con mejores prestaciones. El uso de dichas tabletas supuso la necesidad de dotar a todo el centro de una red WiFi a la que tanto alumnos como profesores pudieran conectarse para desarrollar sus funciones, así como disponer de proyectores o pizarras digitales en todas las aulas.

\subsection{Procedimiento de trabajo}

Una de las características del proyecto EDUCABE consiste en una estructura educativa de centro que proporciona a cada profesor un espacio donde incluir la programación de su asignatura. Dicho espacio se basa en una plataforma creada en Google Drive ${ }^{1}$ y Gesdicodi ${ }^{2}$ que permite acceder al temario, tanto a los alumnos como a sus padres, bien desde sus tabletas, o bien desde el ordenador de su casa, a través de su identificador como alumno del centro. Asimismo, desde el inicio de cada trimestre, disponen de un Itinerario para cada una de las unidades didácticas de las asignaturas, en el que se detalla cómo se planifica cada una de las sesiones y a la vez cómo se evalúa (metodología, recursos, desempeños, competencias evaluadas...). Esta información también puede ser consultada por los padres y sirve como referencia para estar informados del proceso de aprendizaje de sus hijos, al igual que acerca de cualquier tarea o incluso los contenidos que forman parte de la prueba escrita de cada unidad didáctica.

La planificación inicial por itinerarios es elaborada por cada profesor en base al currículum de la asignatura que imparte, y supone una guía de las sesiones destinadas a cada unidad didáctica con su descripción detallada de actividades a realizar (exposiciones, trabajo en grupo, trabajo de investigación...). Dicho itinerario especifica el uso que cada profesor hace de la tableta en el proceso educativo. Durante el desarrollo de las clases, los alumnos, generalmente, se descargan las unidades didácticas en su tableta y así quedan almacenados todos los materiales curriculares necesarios en cada asignatura. Además disponen de una biblioteca con más de 1000 libros de lectura, atlas y las aplicaciones de varios diccionarios y enciclopedias.

También están disponibles una serie de contenidos en forma de libros digitales proporcionados por editoriales. Ello significa que el acceso a todos estos recursos tecnológicos se puede realizar tanto de manera "online", lo que requiere una conexión Internet de la tableta, como de forma autónoma para aquellos casos o situaciones donde no se dispone de tal conexión. En la Tabla 3 se muestra una plantilla de Itinerario cuyos componentes pueden aplicarse a diversas asignaturas tanto de Educación Primaria como Secundaria. 


\begin{tabular}{|c|c|}
\hline Elemento & Descripción \\
\hline Sesión & Identifica el orden en que se realizan las Actividades que forman parte de un tema. \\
\hline Actividad & Describe el trabajo que se lleva a cabo durante cada Sesión. \\
\hline $\begin{array}{l}\text { Método de } \\
\text { trabajo }\end{array}$ & Permite seleccionar al profesor el enfoque docente sobre la Actividad a realizar. \\
\hline Organización & Describe la forma en que se realiza el trabajo de la Actividad por parte de los alumnos. \\
\hline Recursos & Identifica los contenidos y materiales que se utilizarán para el desarrollo de la Actividad. \\
\hline Desempeños & $\begin{array}{l}\text { Se trata de indicadores que permitirán caracterizar el trabajo realizado durante la } \\
\text { Actividad. }\end{array}$ \\
\hline Evaluación & Describe la estrategia utilizada para valorar el trabajo de la Actividad. \\
\hline Calificación & $\begin{array}{c}\text { Indica los mecanismos o criterios que permitan obtener una cuantificación de la nota a } \\
\text { asignar. }\end{array}$ \\
\hline
\end{tabular}

Tabla 3. Elementos de un Itinerario

En este caso, los ejemplos que se utilizan para describir su aplicación proceden de un tema de la asignatura de Ciencias Sociales. Dicho Itinerario se organiza en Sesiones de 55 minutos durante las cuales se realizan ciertas Actividades. Por ejemplo, mostrar algún contenido teórico (p. e. la composición del Sistema Solar) o realizar una tarea práctica ( $p$. e un ejercicio que identifique los planetas del Sistema Solar). Cada Actividad se basa en un Método que indica el enfoque o metodología docente a seguir, ya sea el Estudio de Casos o la Realización de Proyectos (p. e. una maqueta que simule el funcionamiento del Sistema Solar). Asimismo, se podrá definir la forma de Organización basada en el trabajo individual o grupal de los alumnos y establecer los Desempeños como elementos que caracterizan el trabajo asociado a la Actividad. Un posible Desempeño puede consistir en la capacidad para "describir el concepto de planeta" o la habilidad necesaria para llevar a cabo una "búsqueda sobre los cuerpos celestes". La Evaluación de la Actividad se efectúa en base a diferentes estrategias que pueden ir desde la Observación Directa hasta la Valoración de un Proyecto o la realización de una Prueba Escrita.

El modelo de itinerario incorpora, para cada unidad didáctica, una tabla Excel en la que cada profesor valora cada uno de los aspectos que tendrá en cuenta en base a la planificación de la unidad (participación del alumno en el proceso de aprendizaje, escucha activa, presentación de actividades y tareas individuales y grupales,...) junto con el examen de cada unidad, en su caso. Dicha tabla incorpora un sistema de Calificación elaborado por el docente, que define la ponderación sobre la nota final de cada componente asociado a cada actividad, recurso o sesión de trabajo y también al desarrollo de la prueba de evaluación. 
La Figura 2 muestra parte de un ejemplo de itinerario correspondiente al área de Ciencias Sociales en $1 .{ }^{\text {er }}$ curso de la ESO (1. ${ }^{\text {er }}$ trimestre). Dicho itinerario pretende servir de guía a la hora de plantear las posibles actividades, métodos y recursos a utilizar durante un tema de dicha área, en este caso relacionado con la explicación del "Sistema Solar". A partir del planteamiento de tales elementos se podrá observar el aprovechamiento de las tabletas en relación a los mismos.

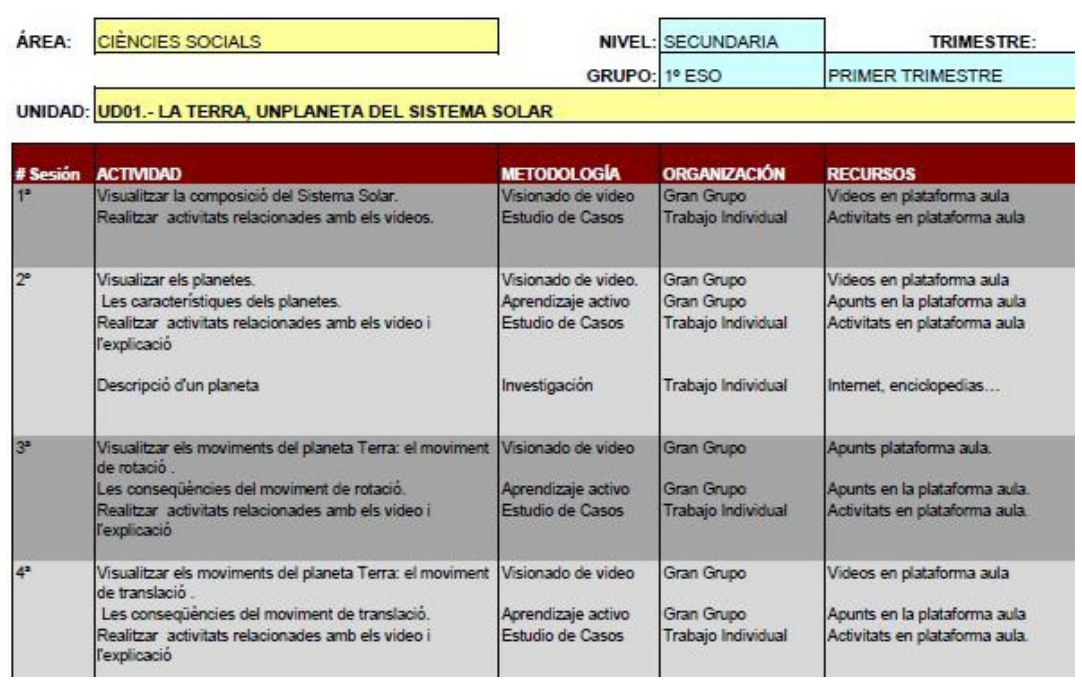

Figura 2. Itinerario sobre Ciencias Sociales en Educación Secundaria

\section{Resultados}

Para la presentación y análisis de los resultados obtenidos se ofrecen dos puntos de vista. Por un lado, se muestran algunos de los resultados académicos en una serie de experiencias basadas en el uso de tabletas y, por otra parte, se exponen los datos de la encuesta realizada durante el curso 201415 para constatar el grado de satisfacción de los usuarios que utilizaron estas.

\subsection{Ejemplos de resultados académicos}

A partir de los elementos expuestos en la anterior sección se describen algunos resultados académicos en asignaturas como Ciencias Sociales. En este caso, una de las pruebas para evaluar la unidad didáctica del sistema solar se basa en el trabajo individual "online". Por ejemplo, las tabletas se usan para responder un cuestionario con preguntas de opción múltiple. Para acceder al mismo, los alumnos deben acreditarse previamente en la plataforma Moodle del centro. Una vez completada la actividad de evaluación, el alumno puede conocer el resultado obtenido y los posibles errores, lo que proporciona una realimentación inmediata. Además, las correspondientes calificaciones se incorporan de forma automática al registro de cada alumno en esa unidad. 
La Figura 3 muestra los resultados del grupo en la prueba "online" con la distribución de notas correspondientes a las respuestas del cuestionario. Como se aprecia existe un porcentaje bastante elevado (cercano al 45\%) de alumnos suspendidos en dicha prueba. Una ventaja en el uso de tecnologías y plataformas como las descritas en este trabajo es la posibilidad de detectar de forma rápida este tipo de situaciones y tomar las acciones pertinentes para su eventual solución.

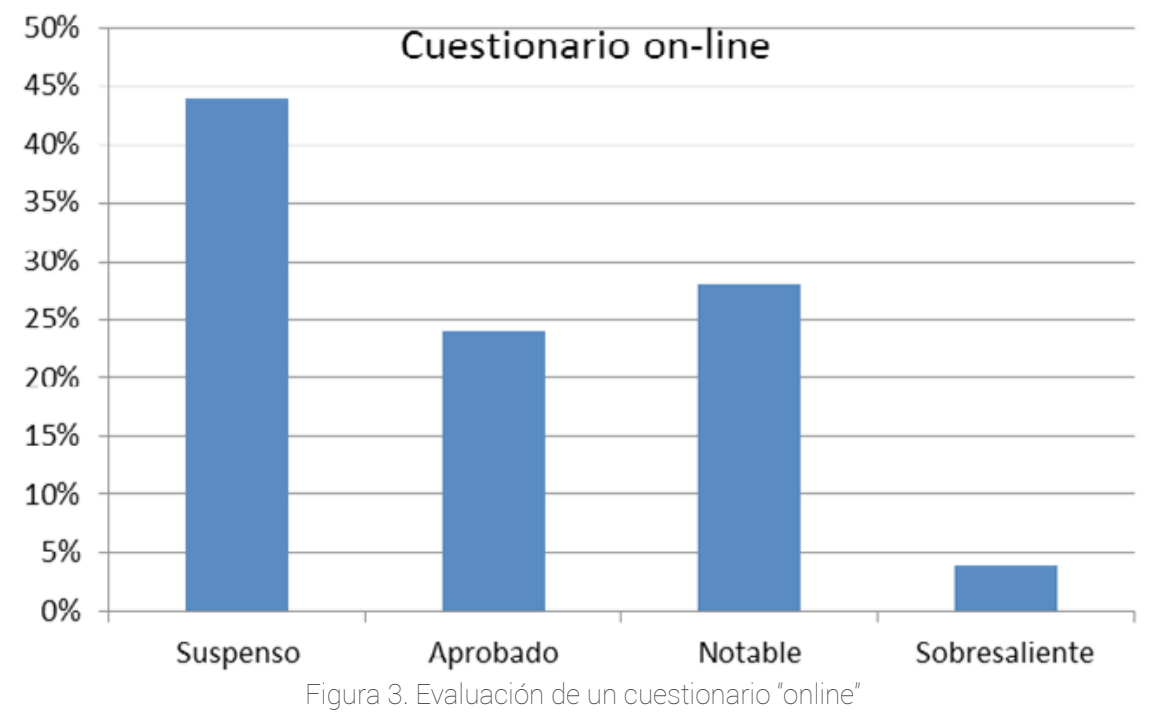

Del mismo modo, la plataforma recoge los resultados obtenidos por cada alumno, a partir de estrategias complementarias empleadas para evaluar la unidad. La Figura 4 muestra la rúbrica utilizada en la evaluación de un informe escrito. Este mecanismo de rúbrica es un aspecto clave en la aplicación didáctica de tecnologías como las tabletas, ya que permite una organización sistemática de las acciones de evaluación. Ello contribuye a facilitar tanto el trabajo del profesor en dicho proceso de medición del rendimiento del alumno como proporcionar a ese mismo alumno los criterios en los que se basará tal evaluación.

Por último, teniendo en cuenta los pesos establecidos en la programación para cada una de las actividades/estrategias de evaluación, se pueden obtener las calificaciones de los alumnos en la unidad didáctica. La Figura 4. muestra los resultados globales obtenidos para la unidad dedicada al sistema solar.

RUBRICA 1: INFORMES ESCRITOS

\begin{tabular}{|c|c|c|c|}
\hline & 4 & 3 & 2 \\
\hline Requisitos & $\begin{array}{l}\text { Cumple con todos los } \\
\text { requisitos. Excede las } \\
\text { expectativas. }\end{array}$ & $\begin{array}{l}\text { Cumple con todos los } \\
\text { requivitos. }\end{array}$ & $\begin{array}{l}\text { No cumple satisfactoria- } \\
\text { mente con algunos } \\
\text { requinitos. }\end{array}$ \\
\hline Exposición del contenido & $\begin{array}{l}\text { Irtormacion presentada } \\
\text { con claridad y muy bien } \\
\text { ofganizada }\end{array}$ & $\begin{array}{l}\text { Información dara y bien } \\
\text { organizada. }\end{array}$ & $\begin{array}{l}\text { Se da informacion, pero } \\
\text { do forma pococ claray } \\
\text { apenas organizada }\end{array}$ \\
\hline Calidad del contenido & $\begin{array}{l}\text { Se ve que se domina el } \\
\text { tema Las partes } \\
\text { principales estan } \\
\text { trabajadas con } \\
\text { argumertos yo } \\
\text { coherencia }\end{array}$ & $\begin{array}{l}\text { Se ve que el tema esta } \\
\text { bastante dominado. La } \\
\text { mayoria de las parnes } \\
\text { estin argumentadas } \% \text { } \\
\text { son coherentes }\end{array}$ & $\begin{array}{l}\text { Es notoria la tala do } \\
\text { seguridad, aunque } \\
\text { aigunas partes estin bien } \\
\text { acgumentadas yo soan } \\
\text { coherentes }\end{array}$ \\
\hline Fuentes & $\begin{array}{l}\text { Se han vinizado todas las } \\
\text { fvertes. }\end{array}$ & $\begin{array}{l}\text { Se han velizizado } 230 \text { de } \\
\text { las fuentes. }\end{array}$ & $\begin{array}{l}\text { Se ha vinizado y/3 de las } \\
\text { fuertes. }\end{array}$ \\
\hline $\begin{array}{l}\text { Reflexión y } \\
\text { reestructuración }\end{array}$ & $\begin{array}{l}\text { Las respuestas han sico } \\
\text { reestructuradas. No so } \\
\text { trata de un corta y pega }\end{array}$ & $\begin{array}{l}\text { Se ve que las respuestas } \\
\text { tienen alguna } \\
\text { reestructuracion, ain } \\
\text { copiar directamente }\end{array}$ & $\begin{array}{l}\text { Las respuestas estän un } \\
\text { poco reestructurados } \\
\text { aunque casi se han } \\
\text { cogiado dinectiamente }\end{array}$ \\
\hline $\begin{array}{l}\text { Presentación det } \\
\text { informe }\end{array}$ & $\begin{array}{l}\text { El informe sipue todas las } \\
\text { normas establecidas para } \\
\text { la presentación de } \\
\text { trabajos. }\end{array}$ & $\begin{array}{l}\text { Pridcticamente se han } \\
\text { seguido todas las normas } \\
\text { de presentacion (2/3) }\end{array}$ & $\begin{array}{l}\text { Al menos se ha } \\
\text { distinguido una portada y } \\
\text { un indice. }\end{array}$ \\
\hline $\begin{array}{l}\text { Ortografia } \\
\text { (dos acentos cuentan } \\
\text { como una falta) }\end{array}$ & $\begin{array}{l}\text { Non hay falas de } \\
\text { othografia ni errores } \\
\text { gramaticales. }\end{array}$ & $\begin{array}{l}\text { Menon de tres takas de } \\
\text { ontografia you errores } \\
\text { gramatcales. }\end{array}$ & $\begin{array}{l}\text { Tres o Cuatito emores de } \\
\text { ontografia yto emroces } \\
\text { gramaticalea. }\end{array}$ \\
\hline
\end{tabular}

Figura 4. Rúbrica utilizada en la evaluación de informes escritos 
Pese al carácter limitado del ejemplo analizado, este muestra cómo el uso integrado de diversas tecnologías, como las tabletas digitales y las plataformas de aprendizaje, favorece sin duda la gestión y supervisión de los desempeños alcanzados por los alumnos en las distintas unidades didácticas, así como una evaluación más pormenorizada y razonada de los mismos.

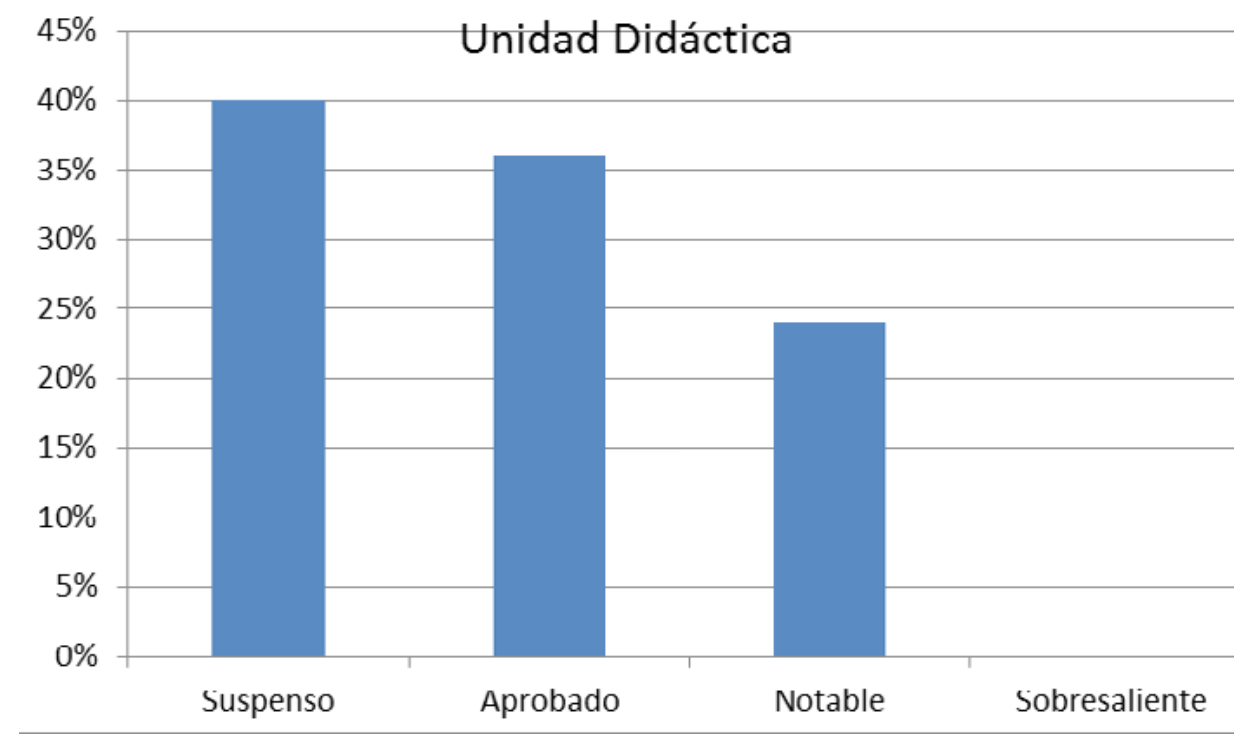

Figura 5. Evaluación de una unidad didáctica

\subsection{Encuestas al alumnado}

Para completar la obtención de resultados se realizó una encuesta al final del curso 2014-15 para evaluar la satisfacción del alumnado de Educación Secundaria respecto a diversos aspectos del trabajo con tabletas. En dicha encuesta se recogieron un total de 129 respuestas (53\% de niñas y $47 \%$ de niños). La distribución de respuestas entre los alumnos encuestados de los diversos cursos de Secundaria fue la siguiente:

- $\quad 1^{\circ} \mathrm{ESO}: 31 \%$

- $\quad 2^{\circ} \mathrm{ESO}: 35 \%$

- $\quad 3^{\circ} \mathrm{ESO}: 21 \%$

- $\quad 4^{\circ} \mathrm{ESO}: 13 \%$

Los datos obtenidos en las respuestas se repartían en una escala Likert con 5 posibles valores desde "Totalmente de acuerdo" a "Totalmente en desacuerdo". La Figura 6 muestra la distribución de respuestas relacionada con la percepción del aprendizaje a partir del uso de tabletas. La circunstancia más llamativa quizás, es el elevado porcentaje de alumnos (superior al 50\%) que consideraban "indiferente" el impacto de tales tecnologías en su nivel de aprendizaje. También llama la atención que menos de un 10\% estuvieran "Totalmente de acuerdo" en su valoración positiva. 


\section{Estoy aprendiendo más [Con la introducción de la Tablet]}

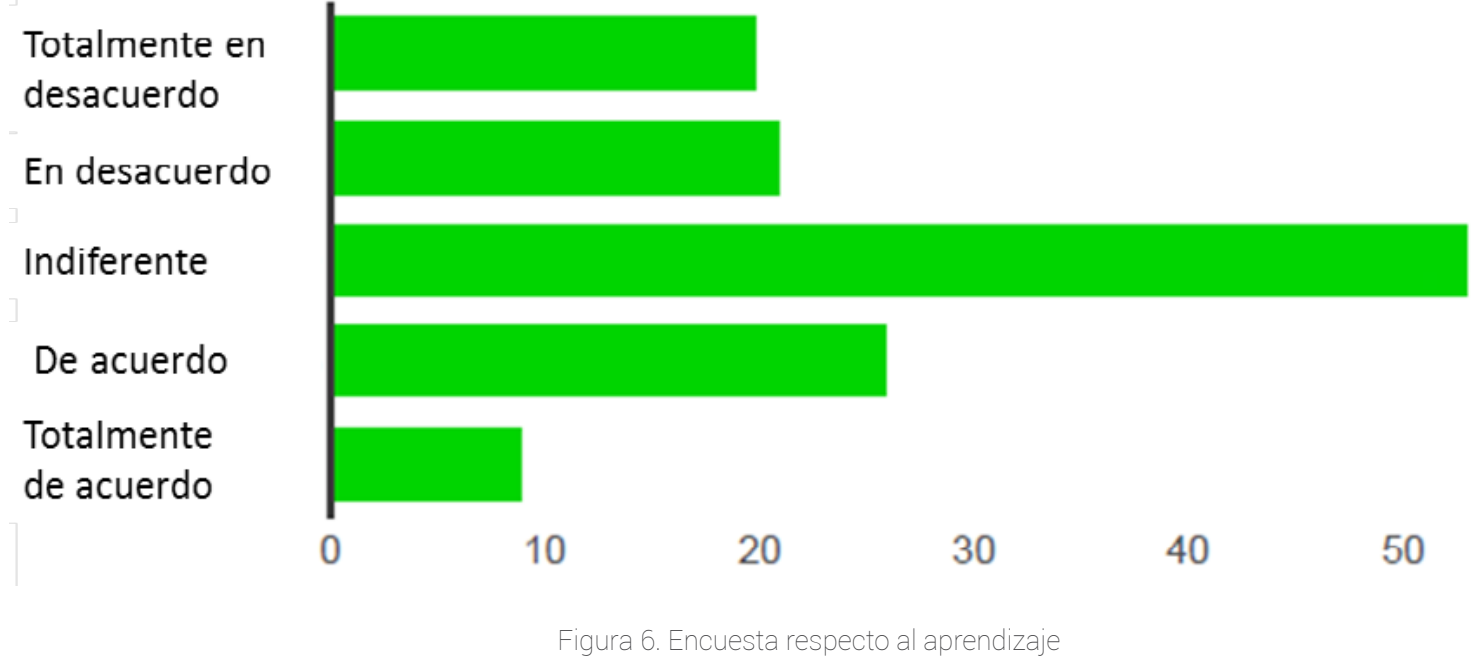

Otra cuestión que se valoró en la encuesta fue la referida a los problemas que se habían detectado en el uso de las tabletas. La Figura 7 reflejaba que uno de los problemas más destacados fue la conexión a Internet junto con la lentitud de la misma. También se detectaron otros problemas técnicos que influyeron en el trabajo con las tabletas.

Asimismo, se recogieron una serie de comentarios realizados al final de la encuesta mediante una pregunta de respuesta abierta. Algunos comentarios destacados consistieron en:

- "Los libros no causan problemas, eran mucho mejor,...".

- "Libros de texto implementados en la tableta, no hechos por los propios profesores, ya que algunos dificultan el estudiar...".

- "Hacer los exámenes en hojas porque en tabletas hay problemas...".

\section{Problemas que tienes o has tenido con la tablet: Señala las opciones correspondientes}

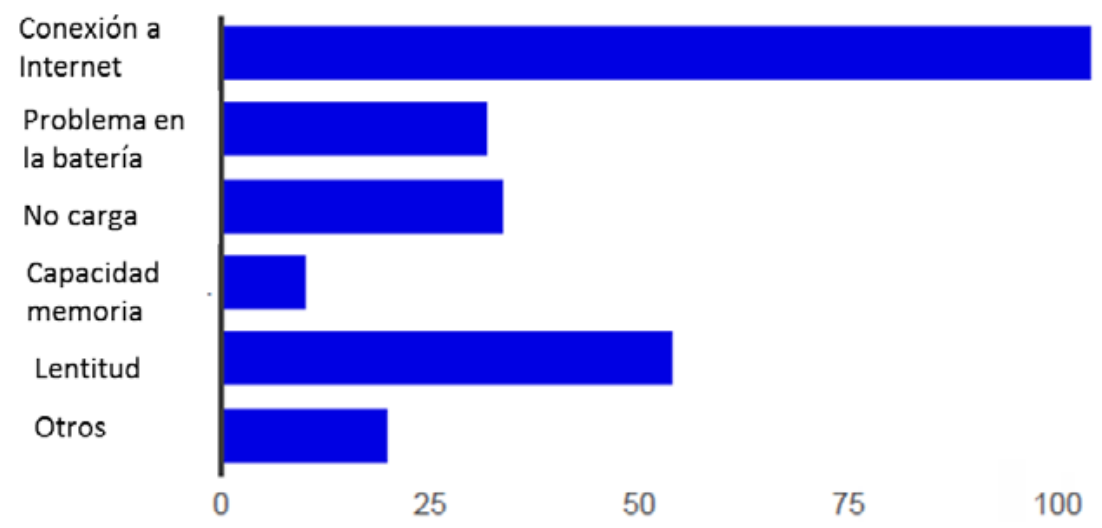


Se trata simplemente de una muestra pero puede servir para llamar la atención sobre las dificultades que perciben los estudiantes a la hora de utilizar las tabletas y la inevitable comparación con los tradicionales libros de texto y métodos de evaluación asociados.

\section{Discusión}

Los resultados mostrados en la sección anterior indican una doble vertiente sobre el aprovechamiento de recursos tecnológicos como las tabletas y su uso en un entorno académico específico. En primer lugar, se hace referencia al seguimiento que puede realizarse de las distintas actividades y pruebas llevadas a cabo en el entorno que nos ocupa. En segundo lugar, se analizan las reacciones de los propios alumnos a la utilización de una tecnología que les plantea diversos desafíos.

Respecto el primer punto, hay que señalar la importancia de una programación estricta que permita organizar las múltiples actividades facilitadas por el uso de las tabletas. Dicha programación resulta necesaria en cualquier contexto académico apoyado en mayor o menor medida por medios tecnológicos, pero es vital cuando la aplicación de este tipo de recursos multiplica las posibilidades de interacción entre alumnos y sus tutores. La definición de Itinerarios, tal como el expuesto en la Figura 2, contribuye a facilitar dicha programación a través de la identificación de Sesiones y las Actividades que se realizan en las mismas, el establecimiento de los Recursos necesarios, o la Evaluación para verificar los Desempeños conseguidos. Esta sería la principal recomendación que pudiera formar parte de una guía sobre la aplicación de recursos tipo tableta en un curso de Secundaria. La improvisación en este tipo de experiencias tecnológicas puede tener un impacto negativo en la evolución del curso dado el espíritu crítico que empieza a arraigar entre los alumnos de esta etapa educativa. Asimismo, la disponibilidad de estos Itinerarios para el profesor que imparte el curso supone una ayuda inestimable para organizar su trabajo día a día. Un segundo aspecto a considerar en esta guía consistiría en la elección de las Actividades a realizar y la Evaluación de estas. En la exposición de Resultados se han descrito las calificaciones obtenidas tras la respuesta a un cuestionario y la evaluación global de una unidad basada en la distribución de valoraciones de las tareas realizadas. En este sentido, la utilización de cuestionarios tipo test o prueba objetiva puede resultar la forma más sencilla de valorar el trabajo realizado por los alumnos. El propio uso de las tabletas facilita en gran medida que dichos cuestionarios puedan ser respondidos de manera rápida y casi en tiempo real durante el transcurso de la propia clase. Sin embargo, existen muchas otras tareas que pueden apoyarse en la utilización de las tabletas, incluso de manera colaborativa entre los alumnos y que fomentan otro tipo de habilidades y competencias. En estos casos, la respuesta a cuestionarios solo representa una parte de la evaluación de tales tareas y se trata de establecer mecanismos de valoración alternativos como pueden ser las rúbricas. La Figura 4. muestra parte de un ejemplo de rúbrica utilizada en la 
evaluación de un informe escrito donde se pueden valorar los distintos aspectos que lo componen, desde cuestiones formales como la inclusión de fuentes bibliográficas o la revisión ortográfica, hasta elementos relacionados con la claridad de presentación o la reflexión en los contenidos introducidos. Por tanto, resulta importante elegir Actividades que puedan fomentar el espíritu creativo de los alumnos o el grado de colaboración entre ellos y donde las tabletas pueden jugar un rol decisivo, pero hay que complementarlas con mecanismos que permitan su Evaluación.

El segundo punto de la Discusión está relacionado con el análisis de las respuestas de una encuesta a alumnos del ciclo de Secundaria a finales del curso 2014-15. En la sección de Resultados se ha expuesto una parte de dichas respuestas en forma de gráficas con los porcentajes de alumnos que manifestaban su opinión en diversos grados de aprobación o desaprobación respecto preguntas como "se aprende más con las tabletas" o "qué problemas has tenido con la tableta". No se expone la encuesta completa, pero resulta bastante significativa en el sentido de detectar cierto grado de desencanto respecto al uso de las tabletas y el impacto que tienen en su aprendizaje. Una parte importante de esta percepción estaría vinculada a los problemas señalados por los propios alumnos en la conexión a Internet o la lentitud en el trabajo con los dispositivos. Pero, si se analizan los comentarios que dichos alumnos adjuntan como complemento a las preguntas de la encuesta, se observa que un alto porcentaje de ellos establece una comparación con experiencias previas basadas en el uso de libros de texto. Hay que tener en cuenta que el perfil de un alumno de Secundaria varía con respecto a la etapa de Primaria, no solo en términos de edad, sino de la procedencia de otros colegios donde se podía trabajar con libros en formato tradicional. Por tanto, es inevitable la comparación entre ambos modos de funcionamiento e imposible subestimar el valor que aportan recursos como los libros de papel. Es innegable que el uso de tabletas en el ámbito de educación Secundaria puede suponer un elemento diferenciador y aportar mejoras a la calidad del proceso de aprendizaje. Sin embargo, hay que valorar si tales dispositivos pueden ser sustitutivos o complemento del tradicional libro de texto. En el primer caso, es preciso estudiar con detalle el perfil de los alumnos que se incorporan a tal experiencia y si tienen los conocimientos y habilidades necesarias para acometer un funcionamiento basado exclusivamente en tabletas. La segunda opción es más conservadora, pero también requiere reflexionar sobre la forma de combinar ambos tipos de recursos y el coste que ello supone. En definitiva, no se puede pensar en una receta "mágica" que resuelva de forma automática todas las cuestiones que introduce el uso de tecnologías como las tabletas y hay que estudiar y diseñar cuidadosamente cada experiencia a realizar. 


\section{Conclusiones}

Este trabajo ha presentado una serie de reflexiones sobre experiencias en el uso de tabletas en el ámbito de la Enseñanza Secundaria. A priori se puede considerar que tales recursos tecnológicos promueven un mayor dinamismo, una mejor interacción entre el alumno y los contenidos, y una adaptación a las nuevas formas de aprendizaje y del conocimiento. También que permiten captar la atención del alumnado facilitando su participación en el proceso de enseñanza, mejorando su memoria visual, su competencia digital y reforzando su creatividad.

Todas estas ventajas no esconden la problemática que acompaña a la utilización de las tabletas y que se traduce en cuestiones como la infraestructura necesaria para su correcto aprovechamiento o la necesidad de formar al profesorado para la aplicación efectiva de este tipo de tecnologías en el aula. En este trabajo se ha incidido en que el uso de estas tecnologías requiere una cuidadosa programación de la secuencia de actividades académicas a realizar, así como una adaptación de los métodos y mecanismos de evaluación a emplear. En caso contrario, pueden surgir situaciones como las detectadas en el caso de estudio, donde se produce un rechazo en el uso de las tabletas a partir de la comparación que realizan los alumnos con una docencia tradicional apoyada en el utilización de recursos clásicos como los libros y las facilidades de uso que este tipo de recursos aportan. Por tanto, es necesario realizar un proceso profundo de reflexión a la hora de introducir estos dispositivos y analizar el valor añadido que supone su uso en los procesos docentes donde se incorporan.

Como futuros trabajos se pretende formalizar todo el proceso de evaluación sobre la aplicación didáctica de las tabletas, tanto a nivel de registro de datos de acceso en el propio dispositivo, como de los resultados almacenados en la plataforma de aprendizaje. Ello incluye la realización de experiencias para poder comparar en determinados grupos la puesta en marcha de actividades específicas en el uso de las tabletas de manera que pueda evaluarse su impacto de manera más precisa.

\section{Referencias}

Arrue, J. (2012). EDUCABE, Educar Bien y en el Bien. Valencia: Colegio Asunción de Benaguasil. Recuperado a partir de: http://asuncionbenaguasil.es/web/pluginfile.php/3416/mod_resource/ content/5/Libro\%20electr\%C3\%B3nico.pdf

CITA (2011). Proyecto Ebook y Educación. Dedos: tabletas digitales en el aula. Salamanca: Centro Internacional de Tecnologías Avanzadas (CITA) en colaboración con la Fundación Germán Sánchez Ruipérez. 
De la Herrán Gascón, A. (2008). Metodología didáctica en Educación Secundaria una perspectiva desde la Didáctica General Metodología didáctica en Educación Secundaria. En A. de la Herrán Gascón \& J. Paredes Labra (Eds.), Didáctica general: la práctica de la enseñanza en Educación Infantil, Primaria y Secundaria. (pp. 134-148) España: McGraw Hill.

Ditendria (2015). Informe Ditendria: Mobile en España y en el Mundo. Madrid: Ditendria Digital Marketing Trends. Recuperado a partir de: http://www.ditrendia.es/wp-content/uploads/2015/07/DitrendiaInforme-Mobile-en-Espa\%C3\%B1a-y-en-el-Mundo-2015.pdf

Ferrer, F., Belvís, E., \& Pàmies, J. (2011). Tablet PCs, academic results and educational inequalities. Computers \& Education, 56(1), 280-288. doi:http://dx.doi.org/10.1016/j.compedu.2010.07.018

Johnson, L., Adams, S. \& Cummins, M. (2012). The NMC Horizon Report: 2012 Higher Education Edition. Austin, Texas: The New Media Consortium. Recuperado a partir de: http://redarchive.nmc.org/ publications/horizon-report-2012-higher-ed-edition

Marés, L. (2012). Tablets en educación. Oportunidades y desafíos en políticas uno a uno. Buenos Aires: Instituto Iberoamericano de TIC y Educación (IBERTIC). Recuperado a partir de: http://www.oei.es/ noticias/spip.php?article10460

Marqués, P. (2014). Metainvestigación sobre el uso educativo de las tabletas digitales. Barcelona: Portal de las tabletas digitales y de los libros de texto digitales. Recuperado a partir de: http://peremarques. net/tabletasportada.htm

UNIR (2013). \#InformeTAB: Estudio sobre el comportamiento de los usuarios de tablet en España. Madrid: Universidad Internacional de la Rioja (UNIR) y Redbility TAB innovation. Recuperado a partir de: http://boletines. prisadigital.com/informe-tab.pdf 